\title{
Uma análise da utilização do método de eliminação de Fourier-Motzkin para encontrar um ponto interior a um poliedro
}

\author{
An analysis of the use of the Fourier-Motzkin elimination method to find an interior point to \\ a polyhedron
}

André Rodrigues Monticeli
Centro Federal de Educação Tecnológica de Minas Gerais (CEFET-MG), Campus Varginha,
Varginha, MG, Brasil
(D) https://orcid.org/0000-0003-4049-5581, andremonticeli@cefetmg.br

Paulo César Mappa

Centro Federal de Educação Tecnológica de Minas Gerais (CEFET-MG), Campus Varginha,

Varginha, MG, Brasil

https://orcid.org/0000-0001-8192-8991, paulomappa@cefetmg.br

\section{Informações do Artigo \\ Como citar este artigo \\ MONTICELI, André Rodrigues; MAPPA, \\ Paulo César. Uma análise da utilização do método de eliminação de Fourier-Motzkin para encontrar um ponto interior a um poliedro. REMAT: Revista Eletrônica da Matemática, Bento Gonçalves, RS, v. 7, n. 1 , p. e3004, 15 jan. 2021. DOI: \\ https://doi.org/10.35819/remat2021v7i1id4305}

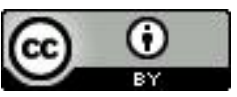

\section{Histórico do Artigo}

Submissão: 2 de julho de 2020.

Aceite: 5 de outubro de 2020 .

\section{Palavras-chave}

Sistemas de Inequações Lineares

Poliedro

Ponto Interior

Eliminação de Fourier-Motzkin

\section{Resumo}

O problema de encontrar um ponto interior a um poliedro tem aplicações em muitas áreas, sobretudo em programação linear. Neste trabalho, abordamos o problema de encontrar um ponto interior a um poliedro, utilizando como estratégia o Método de Eliminação de Fourier-Motzkin. Este método consiste em reduzir um sistema de inequações lineares que define o poliedro, através da eliminação de variáveis. Foi-se utilizada uma versão matricial deste método a fim de facilitar sua implementação computacional e, para ilustrar a metodologia proposta, exemplos são apresentados. Em seguida, fizemos a análise de complexidade do algoritmo, com a finalidade de investigar o comportamento da técnica quando do aumento do número de variáveis e de restrições e, dessa forma, apresentando um campo de aplicação da técnica. Pela análise do algoritmo, concluímos que este tem complexidade exponencial, pois o número de inequações cresce exponencialmente conforme se aumenta o número de variáveis no problema. $O$ algoritmo se mostrou eficiente para problemas com um número pequeno de inequações para o $R^{2}$ e o $R^{3}$.
Keywords

Systems of Linear Inequalities

Polyhedron

Interior Point

Fourier-Motzkin Elimination

\begin{abstract}
The issue of finding an interior point to a polyhedron has applications in many areas, especially in linear programming. In this work we approach the issue of finding an interior point to a polyhedron using the Fourier-Motzkin Elimination Method approach. It consists of reducing a system of linear inequalities that defines the polyhedron, by eliminating variables. A matrix version of this method was employed in order to facilitate its computational implementation, and some examples were presented with the purpose of illustrating the proposed methodology. Subsequently the complexity analysis of the algorithm was carried out in order to investigate the behavior of the technique when increasing the number of variables and restrictions and, thus, presenting a field of application to the technique. By analyzing the algorithm, we concluded that it has exponential complexity, since the number of inequalities grows exponentially as the number of variables in the issue increases. The algorithm proved to be efficient for issue with a small number of inequalities for $R^{2}$ and $R^{3}$.
\end{abstract}




\section{Introdução}

Um sistema de inequações lineares é um conjunto de inequações lineares nas mesmas variáveis, conforme Equação 1.

$$
\left\{\begin{array}{c}
a_{11} x_{1}+a_{12} x_{2}+\ldots+a_{1 n} x_{n} \leq b_{1} \\
a_{21} x_{1}+a_{22} x_{2}+\ldots+a_{2 n} x_{n} \leq b_{2} \\
\vdots \\
a_{m 1} x_{1}+a_{m 2} x_{2}+\ldots+a_{m n} x_{n} \leq b_{m}
\end{array}\right.
$$

onde $x_{1}, x_{2}, \ldots, x_{n}$ são as variáveis, $a_{11}, a_{12}, \ldots a_{m n}$ são os coeficientes lineares do sistema de inequações e $b_{1}, b_{2}, \ldots, b_{m}$ são os termos constantes.

Podemos escrever a Equação 1 na forma matricial, conforme Equação 2.

$$
A x \leq b
$$

onde

$$
A=\left[\begin{array}{cccc}
a_{11} & a_{12} & \cdots & a_{1 n} \\
a_{21} & a_{22} & \cdots & a_{2 n} \\
\vdots & \cdots & \vdots \\
a_{m 1} & a_{m 2} & \cdots & a_{m n}
\end{array}\right], \quad b=\left[\begin{array}{c}
b_{1} \\
b_{2} \\
\vdots \\
b_{m}
\end{array}\right] \text { e } \quad x=\left[\begin{array}{c}
x_{1} \\
x_{2} \\
\vdots \\
x_{n}
\end{array}\right]
$$

A matriz $A_{m \times n}$ é denominada matriz dos coeficientes, $x$ é denominado vetor das variáveis e $b$ é denominado vetor dos termos independentes.

A região de solução de um sistema de inequações lineares é chamada de poliedro. Quando esta região for limitada, dizemos que o poliedro é um politopo (LAURITZEN, 2009).

O problema de encontrar um ponto interior a um poliedro tem aplicações em muitas áreas, sobretudo em programação linear e, em geral, é considerado como um problema difícil. Existem vários trabalhos dedicados exclusivamente a esta questão, por exemplo Cartis e Nicholas (2009), Barvinok e Pommersheim (1999) e Cui et al. (2011).

Um ponto $c$ é dito interior a um poliedro, ou seja, interior de um sistema de inequações $A x \leq b$ com $m$ inequações se $a_{i}^{T} c<b_{i}$ para todo $i=1,2, \ldots, m$.

Monticeli (2010) descreve e aplica a Eliminação de Fourier-Motzkin na obtenção de um ponto interior a um poliedro por meio da eliminação de variáveis de um sistema de inequações lineares, que definem o poliedro; todavia, não se preocupa em investigar limites para sua aplicação. No presente trabalho, apresentamos um algoritmo baseado em uma formulação matricial para a obtenção de um ponto interior pelo Método Eliminação de Fourier-Motzkin e, por meio da análise de complexidade, estabelecemos o campo de aplicabilidade da técnica, deixando claro onde a técnica é eficiente e qual custo computacional esperar de seu uso.

Doravante, sempre que nos referirmos a um ponto interior, estaremos tratando do ponto interior ao poliedro definido pelo sistema de inequações. A técnica consiste em, a partir do sistema 
original em $R^{n}$, obter um dual em $R^{n-1}$ cuja solução é a projeção da solução do sistema original sobre $R^{n-1} \mathrm{e}$, assim, sucessivamente, chegar à projeção da solução em $R$ para, através de uma retro substituição, solucionar o problema original. Para a avaliação da técnica, foi feito a análise da complexidade de tempo, número de operações realizadas; e a complexidade de espaço, memória necessária em sua execução (DEMMEL, 1997).

Este artigo está organizado da seguinte forma: na seção 2, apresentamos de maneira formal o Método de Eliminação de Fourier-Motzkin. No final desta seção, apresentamos alguns resultados em que, por meio do Método de Eliminação de Fourier-Motzkin, é possível verificar se o sistema de inequações tem, ou não, solução e se a região de solução é limitada. Estes resultados poderão ser encontrados em Dahl (2007). Na seção 3, trabalhamos o método por meio de operações com matrizes, o que facilitará sua abordagem computacional. Na seção 4, utilizaremos o método de Fourier-Motzkin para obtenção de um ponto interior a um poliedro em exemplos. $\mathrm{Na}$ seção 5, apresentaremos a complexidade do algoritmo e alguns testes computacionais. E, na última seção, algumas considerações finais.

\section{O método de eliminação de Fourier-Motzkin}

Nesta seção, vamos apresentar o método de eliminação de Fourier-Motzkin seguindo as ideias vistas em Monticeli (2010) e Monticeli e Torezzan (2010).

A eliminação de Fourier-Motzkin é o método mais antigo para resolver um sistema de inequações lineares. Foi descoberto em 1827 por Joseph Fourier (Fourier, 1890). O método foi descrito na tese de doutorado de Motzkin em 1983 (MOTZKIN, 1983).

Seja o sistema de inequações lineares $A x \leq b$, representado conforme Equação 1.

Para cada $i$, onde $a_{i 1} \neq 0$, podemos multiplicar cada uma das inequações por $\frac{1}{\left|a_{\mathrm{i} 1}\right|}$, deixando como valor do coeficiente de $x_{1}, 0$ ou \pm 1 .

Temos, então, um novo sistema de inequações lineares, conforme Equação 4,

$$
\left\{\begin{array}{r}
-x_{1}+a_{i 2}^{\prime} x_{2}+\ldots+a_{i n}^{\prime} x_{n} \leq b_{i}^{\prime} \quad\left(i \in I^{-}\right) \\
x_{1}+a_{i 2}^{\prime} x_{2}+\ldots+a_{i n}^{\prime} x_{n} \leq b_{i}^{\prime} \quad\left(i \in I^{+}\right) \\
a_{i 2}^{\prime} x_{2}+\ldots+a_{i n}^{\prime} x_{n} \leq b_{i}^{\prime} \quad\left(i \in I^{0}\right)
\end{array}\right.
$$

onde $I^{-}=\left(i: a_{i 1}<0\right)$, ou seja, as inequações onde os coeficientes de $x_{1}$ são negativos, $I^{+}=$ $\left(i: a_{i 1}>0\right)$, ou seja, as inequações onde os coeficientes de $x_{1}$ são positivos e $I^{0}=\left(i: a_{i 1}=0\right)$, ou seja, as inequações onde os coeficientes de $x_{1}$ são nulos, $a_{i j}^{\prime}=\frac{a_{i j}}{\left|a_{i 1}\right|}$ e $b_{i}^{\prime}=\frac{b_{i}}{\left|a_{i 1}\right|}$.

Assim, o conjunto de índices da linha $I=\{1,2, \ldots, m\}$ é particionado nos subconjuntos $I^{-}, I^{+}$ e $I^{0}$, sendo que algum destes pode ser vazio. Daqui resulta que $x_{1}, x_{2}, x_{3}, \ldots, x_{n}$ é uma solução do 
sistema original conforme Equação 1 se, e somente se, $x_{1}, x_{2}, x_{3}, \ldots, x_{n}$ satisfizer as restrições, conforme Equação 5 e Equação 6.

$$
\max \left\{\left(a_{k 2}^{\prime} x_{2}+\ldots+a_{k n}^{\prime} x_{n}\right)-b_{k}^{\prime}\right\} \leq x_{1} \leq \min \left\{b_{i}^{\prime}-\left(a_{i 2}^{\prime} x_{2}+\ldots+a_{i n}^{\prime} x_{n}\right)\right\}
$$

onde $i \in I^{+}, k \in I^{-} \mathrm{e}$

$$
a_{i 2}^{\prime} x_{2}+a_{i 3}^{\prime} x_{3}+\ldots+a_{i n}^{\prime} x_{n} \leq b_{i}^{\prime}
$$

para o coeficiente de $x_{1}$ valendo zero, com $i \in I^{0}$.

A partir da Equação 5 , temos que $x_{1}$ encontra-se em um certo intervalo, que é determinado por $x_{2}, x_{3}, \ldots, x_{n}$. Assim, podemos escrever a Equação 7

$$
\max \left\{\left(a_{k 2}^{\prime} x_{2}+\ldots+a_{k n}^{\prime} x_{n}\right)-b_{k}^{\prime}\right\} \leq \min \left\{b_{i}^{\prime}-\left(a_{i 2}^{\prime} x_{2}+\ldots+a_{i n}^{\prime} x_{n}\right)\right\}
$$

que pode ser resolvida combinando cada inequação do primeiro conjunto de restrições com cada inequação do segundo conjunto de restrições.

Juntando os resultados obtidos, ao resolver a Equação 7 com a Equação 6, obtemos a projeção, Proj, no espaço das variáveis $x_{2}, x_{3}, \ldots, x_{n}$.

Pode-se, então, proceder da mesma forma e eliminar as demais variáveis.

A partir do método de eliminação de Fourier-Motzkin temos as seguintes observações, conforme Dahl (2007):

- Se $I^{-}$ou $I^{+}$for vazio, o primeiro conjunto de restrições da Equação 5 desaparece e o sistema será inconsistente ou terá uma região ilimitada, pois a variável $x_{1}$ não terá limite superior $\left(I^{-}=\varnothing\right)$ ou não terá limite inferior $\left(I^{+}=\emptyset\right)$.

- Se o conjunto $I^{0}$ for vazio e apenas um dos conjuntos $I^{-}$ou $I^{+}$for não vazio, não podemos eliminar a primeira variável. Uma alternativa é reordenar o sistema e iniciar a eliminação por outra variável.

- O método de eliminação de Fourier-Motzkin nos permite concluir se o sistema é inconsistente ou não. Para isto, basta eliminar todas as variáveis do sistema formando um sistema de desigualdade constantes.

\section{Eliminação de Fourier-Motzkin por meio de operações com matrizes}

Podemos utilizar a notação matricial para apresentar o método de eliminação de FourieVírMotzkin de outro ponto de vista, conforme descrito no trabalho de Dahl (2007). A seguir, descreveremos, de forma resumida esta abordagem, como está apresentada em Monticeli e Torezzan (2010).

Considere o sistema linear, conforme Equação 1, e sua matriz de coeficientes $A \in R^{m \times n}$. Seja $A^{\prime}$ a matriz dos coeficientes de um sistema linear ajustado, de acordo com o sistema linear 
da Equação 2, ainda com todas as variáveis $\mathrm{x}_{1}, \mathrm{x}_{2}, \ldots, \mathrm{x}_{\mathrm{n}}$, mas cujos coeficientes de $x_{1}$ são 0 ou \pm 1 . O sistema de inequações lineares pode ser representado conforme Equação 8.

$$
A^{\prime} x \leq b^{\prime}
$$

ou ainda,

$$
\left[\begin{array}{cccc}
a_{11}^{\prime} & a_{12}^{\prime} & \cdots & a_{1 n}^{\prime} \\
a_{21}^{\prime} & a_{22}^{\prime} & \cdots & a_{2 n}^{\prime} \\
\vdots & \vdots & \cdots & \vdots \\
a_{m 1}^{\prime} & a_{m 2}^{\prime} & \cdots & a_{m n}^{\prime}
\end{array}\right] \cdot\left[\begin{array}{c}
x_{1} \\
x_{2} \\
\vdots \\
x_{n}
\end{array}\right] \leq\left[\begin{array}{c}
b_{1}^{\prime} \\
b_{2}^{\prime} \\
\vdots \\
b_{m}^{\prime}
\end{array}\right]
$$

Agora vamos criar uma matriz positiva $C \in R^{m^{\prime} \times m}$, onde $m^{\prime}=\left|I^{+}\right| \times\left|I^{-}\right|+\left|I^{0}\right|$, ou seja, o número de linhas da matriz $C$ é o produto entre o número de coeficientes $a_{i 1}^{\prime}>0$ e $a_{i 1}^{\prime}<0$ mais o número de coeficientes $a_{i 1}^{\prime}=0$, e o número de colunas, $m$, é igual ao número de linhas da matriz $A^{\prime}$.

A matriz $C$ será uma matriz binária (seus elementos serão 0 ou 1) de forma a representar as combinações entre o conjunto das inequações que satisfazem $a_{i 1}^{\prime}<0$ com aquelas que satisfazem $a^{\prime}{ }_{i 1}>0$. Vamos supor que as linhas $a_{1}^{\prime}, a_{2}^{\prime}, \ldots, a_{p}^{\prime}$ são aquelas onde $a_{i 1}^{\prime}<0$, as linhas $a_{p+1}^{\prime}, a_{p+2}^{\prime}, \ldots, a_{p+r}^{\prime}$ são aquelas onde $a_{i 1}^{\prime}>0$ e $a_{p+r+1}^{\prime}, a_{p+r+2}^{\prime}, \ldots, a_{m}^{\prime}$ as linhas que satisfazem a ${ }^{\prime}{ }_{11}=$ 0 . Neste caso, a matriz $C$ terá a forma apresentada na Equação 10.

$C=$\begin{tabular}{cccc|cccc|cccc}
1 & 0 & $\cdots$ & 0 & 1 & 0 & $\cdots$ & 0 & 0 & 0 & $\cdots$ & 0 \\
1 & 0 & $\cdots$ & 0 & 0 & 1 & $\cdots$ & 0 & 0 & 0 & $\cdots$ & 0 \\
1 & 0 & $\cdots$ & 0 & 0 & 0 & $\cdots$ & 1 & 0 & 0 & $\cdots$ & 0 \\
\hline$\vdots$ & $\vdots$ & $\cdots$ & $\vdots$ & $\vdots$ & $\vdots$ & $\cdots$ & $\vdots$ & $\vdots$ & $\vdots$ & $\cdots$ & 0 \\
\hline 0 & 0 & $\cdots$ & 1 & 1 & 0 & $\cdots$ & 0 & 0 & 0 & $\cdots$ & 0 \\
0 & 0 & $\cdots$ & 1 & 0 & 1 & $\cdots$ & 0 & 0 & 0 & $\cdots$ & 0 \\
0 & 0 & $\cdots$ & 1 & 0 & 0 & $\cdots$ & 1 & 0 & 0 & $\cdots$ & 0 \\
\hline$\vdots$ & $\vdots$ & $\cdots$ & $\vdots$ & $\vdots$ & $\vdots$ & $\cdots$ & $\vdots$ & $\vdots$ & $\vdots$ & $\cdots$ & 0 \\
\hline 0 & 0 & $\cdots$ & 0 & 0 & 0 & $\cdots$ & 0 & 1 & 0 & $\cdots$ & 0 \\
0 & 0 & $\cdots$ & 0 & 0 & 0 & $\cdots$ & 0 & 0 & 1 & $\cdots$ & 0 \\
$\vdots$ & $\vdots$ & $\cdots$ & $\vdots$ & $\vdots$ & $\vdots$ & $\cdots$ & $\vdots$ & $\vdots$ & $\cdots$ & $\ddots$ & 0 \\
0 & 0 & $\cdots$ & 0 & 0 & 0 & $\cdots$ & 0 & 0 & 0 & $\cdots$ & 1
\end{tabular}

Com a matriz $C$ construída, para eliminar a primeira variável, basta efetuar as operações com matrizes conforme Equação 11.

$$
C \times A^{\prime} \text { e } C \times b^{\prime}
$$

Assim, construímos a seguinte desigualdade: 


$$
\left[\begin{array}{cccc}
0 & a^{\prime \prime}{ }_{12} & \cdots & a^{\prime \prime}{ }_{1 n} \\
0 & a_{22} & \cdots & a^{\prime \prime}{ }_{2 n} \\
\vdots & \vdots & \cdots & \vdots \\
0 & a^{\prime \prime}{ }_{m^{\prime} 2} & \cdots & a^{\prime \prime}{ }_{m_{n}}
\end{array}\right] \cdot\left[\begin{array}{c}
x_{1} \\
x_{2} \\
\vdots \\
x_{n}
\end{array}\right] \leq\left[\begin{array}{c}
b^{\prime \prime}{ }_{1} \\
b{ }_{2} \\
\vdots \\
b^{\prime \prime}{ }_{m^{\prime}}
\end{array}\right]
$$

onde $a^{\prime \prime}{ }_{m^{\prime} i}$ são os resultados da operação $C \times A^{\prime}$ e $b^{\prime \prime} m^{\prime}$ são os resultados da operação $C \times b^{\prime}$. A variável $x_{1}$ pode ser eliminada deletando a primeira coluna da matriz $C \times A^{\prime}$. Assim, podemos proceder da mesma forma e eliminar as variáveis $x_{2}, x_{3}$, etc.

\section{Obtendo um ponto interior a um poliedro pelo método de eliminação de Fourier- Motzkin}

Para encontrar um ponto interior a um poliedro utilizando o método de eliminação de Fourier-Motzkin, escolhemos um eixo para projetar a solução, encontrando seus pontos extremos, depois obtemos o ponto médio e calculamos as coordenadas correspondentes a este ponto escolhido. Se, ao projetarmos, não existir limite superior, escolhemos como o ponto pertencente à projeção o ponto resultante do limite_inferior $+k$ sendo $k$ um escalar. Se não existir um limite inferior, o ponto escolhido será limite_superior - $k$. Caso não exista limite inferior e nem superior, iremos escolher o zero. A seguir, apresentaremos duas aplicações para ilustrar este processo.

\subsection{Aplicação 1}

Consideremos o sistema de inequações lineares Equação 13, cuja região de solução está apresentada na Figura 1.

$$
\begin{aligned}
z & \leq 4 \\
-z & \leq-2 \\
4 x+z & \leq 10 \\
-4 x-z & \leq 0 \\
-2 y+z & \leq 0 \\
2 y-z & \leq 4
\end{aligned}
$$


Figura 1 - Poliedro solução do sistema de inequações lineares da Aplicação 1.

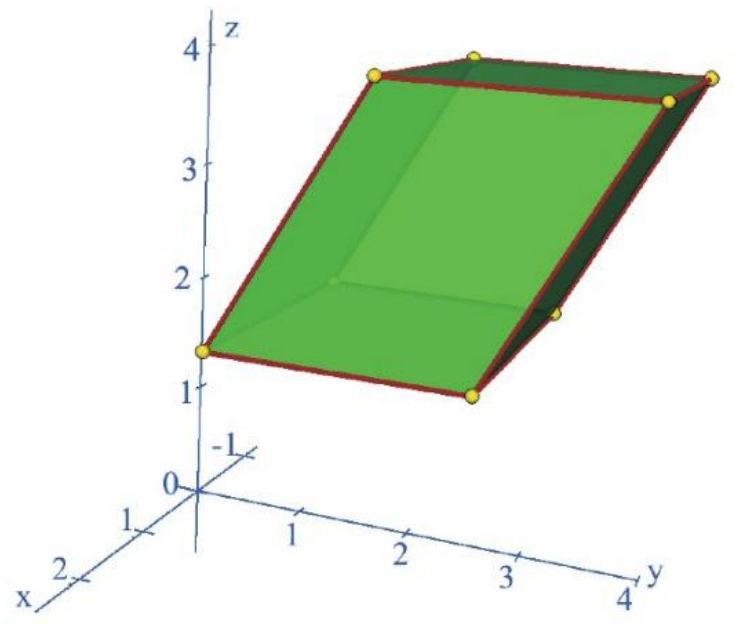

Fonte: Monticeli (2010, p. 48).

Vamos eliminar a variável $z$, projetando no plano $x y$ a região de solução do sistema linear, Equação 13. Para isso, vamos rearranjá-lo, reescrevendo-o de tal forma que a variável a ser eliminada, variável z, esteja no início de cada inequação. Além disso, organizamos as inequações do sistema, colocando primeiro as que possuem coeficientes negativos, seguido das que possuem coeficientes positivos e, caso existam, os coeficientes zero. Assim temos,

$$
\begin{aligned}
-z & \leq-2 \\
-z-4 x & \leq 0 \\
-z+2 y & \leq 4 \\
z & \leq 4 \\
z+4 x & \leq 10 \\
z-2 y & \leq 0
\end{aligned}
$$

Agora, como os coeficientes da variável a ser eliminada, $z$, já são 0 ou \pm 1 , podemos escrever as matrizes $A$ e b correspondentes ao sistema de inequações, Equação 14, e determinar a matriz $C$. Assim temos,

$$
A=\left[\begin{array}{ccc}
-1 & 0 & 0 \\
-1 & -4 & 0 \\
-1 & 0 & 2 \\
1 & 0 & 0 \\
1 & 4 & 0 \\
1 & 0 & -2
\end{array}\right], \quad b=\left[\begin{array}{c}
-2 \\
0 \\
4 \\
4 \\
10 \\
0
\end{array}\right]
$$

A matriz $C$ terá: 6 colunas e $(3 \times 3)+0=9$ linhas. Assim, determinamos a matriz $C$, conforme Equação 16. 


$C=$\begin{tabular}{lll|lll}
1 & 0 & 0 & 1 & 0 & 0 \\
1 & 0 & 0 & 0 & 1 & 0 \\
1 & 0 & 0 & 0 & 0 & 1 \\
\hline 0 & 1 & 0 & 1 & 0 & 0 \\
0 & 1 & 0 & 0 & 1 & 0 \\
0 & 1 & 0 & 0 & 0 & 1 \\
\hline 0 & 0 & 1 & 1 & 0 & 0 \\
0 & 0 & 1 & 0 & 1 & 0 \\
0 & 0 & 1 & 0 & 0 & 1
\end{tabular}

Efetuando as operações $C \times A$ e $C \times b$ temos,

$$
C \times A=\left[\begin{array}{ccc}
0 & 0 & 0 \\
0 & 4 & 0 \\
0 & 0 & -2 \\
0 & -4 & 0 \\
0 & 0 & 0 \\
0 & -4 & -2 \\
0 & 0 & 2 \\
0 & 4 & 2 \\
0 & 0 & 0
\end{array}\right], \quad C \times b=\left[\begin{array}{c}
2 \\
8 \\
-2 \\
4 \\
10 \\
0 \\
8 \\
14 \\
4
\end{array}\right]
$$

Desta forma, eliminamos a primeira coluna da matriz $C \times A$, e obtemos um novo sistema de inequações lineares sem a variável $z$.

$$
\begin{aligned}
0 & \leq 2 \\
4 x & \leq 8 \\
-2 y & \leq-2 \\
-4 x & \leq 4 \\
0 & \leq 10 \\
-4 x-2 y & \leq 0 \\
2 y & \leq 8 \\
4 x+2 y & \leq 14 \\
0 & \leq 4
\end{aligned}
$$

Na Figura 2 temos a região de solução projetada no plano $x y$.

Figura 2 - Projeção da região de solução do sistema no plano $x y$.

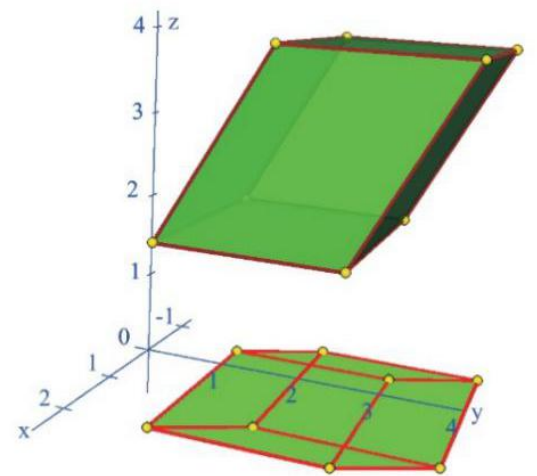

Fonte: Monticeli (2010, p. 50). 
Ao eliminar a variável $y$, temos os limites para a variável $x$. O sistema de inequações encontrado somente na variável $x$ está apresentado na Equação 19.

$\begin{aligned} 0 & \leq 3 \\ 2 x & \leq 6 \\ -2 x & \leq 4 \\ 0 & \leq 7 \\ 4 x & \leq 8 \\ -4 x & \leq 4 \\ 0 & \leq 2 \\ 0 & \leq 10 \\ 0 & \leq 4\end{aligned}$

Portanto, os valores de $x$ que satisfazem todas as inequações estão compreendidos entre -1 e 2 . Assim, determinamos o valor de $x$, conforme Equação 20.

$$
\overline{\mathrm{x}}=\frac{-1+2}{2}=\frac{1}{2}
$$

Substituindo o valor de $\bar{x}=\frac{1}{2}$ no sistema da Equação 18 , obtemos os limites de $y$, sendo $1 \leq y \leq 4$. Assim, o valor de $y$ correspondente à $\bar{x}=\frac{1}{2}$ é dado pela Equação 21 .

$$
\bar{y}=\frac{1+4}{2}=\frac{5}{2}
$$

Desta forma, para obtermos o ponto interior, substituiremos os valores $\bar{x}=\frac{1}{2}$ e $\bar{y}=\frac{5}{2}$ no sistema Equação 14 encontrando o valor de $z$ correspondente.

$$
\bar{z}=\frac{2+4}{2}=3
$$

Portanto, um ponto interior ao poliedro é $p=\left(\frac{1}{2}, \frac{5}{2}, 3\right)$.

\subsection{Aplicação 2}

Consideremos o seguinte sistema de inequações lineares, cuja região é um poliedro do $R^{4}$.

$$
\left\{\begin{aligned}
0,7071 x_{1}+0,4082 x_{2}+0,2887 x_{3}+0,5 x_{4} & \leq 3,4654 \\
-0,7071 x_{1}-0,4082 x_{2}-0,2887 x_{3}-0,5 x_{4} & \leq-1,4654 \\
-0,7071 x_{1}+0,4082 x_{2}+0,2887 x_{3}+0,5 x_{4} & \leq-0,7772 \\
0,7071 x_{1}-0,4082 x_{2}-0,2887 x_{3}-0,5 x_{4} & \leq 2,7772 \\
-0,8164 x_{2}+0,2887 x_{3}+0,5 x_{4} & \leq-3,5543 \\
0,8164 x_{2}-0,2887 x_{3}-0,5 x_{4} & \leq 5,5547 \\
-0,8660 x_{3}+0,5 x_{4} & \leq 0,8660 \\
0,8660 x_{3}-0,5 x_{4} & \leq 1,1340
\end{aligned}\right.
$$


Aplicando o algoritmo de ponto interior por Fourier-Motzkin, obtemos o seguinte ponto interior ao poliedro $(p)$.

$$
p=(3,3.999,-1,-2)
$$

\section{Análise do algoritmo}

Fazer a análise do algoritmo é analisar sua complexidade computacional, ou seja, predizer os recursos computacionais que o algoritmo requer quando da sua execução. Neste estudo, vamos fazer a análise da complexidade de tempo e da complexidade de espaço.

Para analisar a complexidade de tempo e a complexidade de espaço do algoritmo, considere o problema geral com $m$ inequações e $n$ variáveis e o pior dos casos, ou seja, $\left|I^{+}\right|=$ $\left|I^{-}\right|$e $\left|I^{0}\right|=0$, além de que não há inequações redundantes. Então, no sistema $A x \leq b$, temos as matrizes $A_{m \times n}$ e $b_{m \times 1}$.

Eliminando uma variável, $x_{1}$, no pior dos casos teremos $\left|I^{+}\right|=\frac{m}{2}$ e $\left|I^{-}\right|=\frac{m}{2}$, e , assim, a dimensão da matriz $C$ e das operações $C \times A$ e $C \times b$ são dadas pela Equação 25, Equação 26 e Equação 27.

$$
\begin{gathered}
C_{\frac{m^{2}}{4} \times m} \\
C_{\frac{m^{2}}{4} \times m} \cdot A_{m \times n}=A_{\frac{m^{2}}{4} \times n}^{I} \rightarrow A_{\frac{m^{2}}{4} \times(n-1)}^{I^{\prime}} \\
C_{\frac{m^{2}}{4} \times m} \cdot b_{m \times 1}=b_{\frac{m^{2}}{4} \times 1}^{I}
\end{gathered}
$$

Assim, temos um novo sistema $A^{I^{\prime}} x^{I} \leq b^{I}$ sem a variável $x_{1}$. Aplicando o mesmo procedimento para eliminar uma segunda variável, $x_{2}$, temos, no pior dos casos $\left|I^{+}\right|=\left|I^{-}\right|=$ $\frac{m^{2}}{4} \cdot \frac{1}{2}=\frac{m^{2}}{8}$.

$$
\begin{aligned}
& C_{\frac{m^{4}}{64} \times \frac{m^{2}}{4}}^{I} \\
& C_{\frac{m^{4}}{64} \times \frac{m^{2}}{4}}^{I} \cdot A_{\frac{m^{2}}{4} \times(n-1)}^{I^{\prime}}=A_{\frac{m^{4}}{64} \times(n-1)}^{I I} \rightarrow A_{\frac{m^{4}}{64} \times(n-2)}^{I I^{\prime}}
\end{aligned}
$$




$$
C_{\frac{m^{4}}{64} \times \frac{m^{2}}{4}}^{I} \cdot b_{\frac{m^{2}}{4} \times 1}^{I}=b_{\frac{m^{4}}{64} \times 1}^{I I}
$$

Assim, temos um novo sistema $A^{I I^{\prime}} x^{I I} \leq b^{I I}$ sem as variáveis $x_{1}$ e $x_{2}$. Aplicando o mesmo procedimento para eliminar uma terceira variável, $x_{3}$, temos, no pior dos casos, $\left|I^{+}\right|=\left|I^{-}\right|=$ $\frac{m^{4}}{64} \cdot \frac{1}{2}=\frac{m^{4}}{128}$.

$$
\begin{gathered}
C_{\frac{m^{8}}{16384} \times \frac{m^{4}}{64}}^{I I}=A_{\frac{m^{8}}{11 I} \times(n-2)}^{\rightarrow} \rightarrow A^{I I I^{\prime}} \frac{m^{8}}{16384} \times(n-3) \\
\frac{C^{8}}{16384} \times \frac{m^{4}}{64} \cdot A_{\frac{m^{4}}{64} \times(n-2)}^{I I^{\prime}} \times C_{\frac{m^{8}}{16384} \times \frac{m^{4}}{64} \cdot b_{\frac{m^{4}}{64} \times 1}^{I I}=b_{\frac{m^{8}}{16384} \times 1}^{I I}}
\end{gathered}
$$

Assim, o novo sistema $A^{I I I} x^{I I I} \leq b^{I I I}$ estará sem as variáveis $x_{1}, x_{2}$ e $x_{3}$. Podemos seguir este procedimento eliminando as demais variáveis do sistema ao deixar apenas uma.

Para a complexidade de tempo do algoritmo temos que, para cada eliminação de uma variável, as operações relevantes são duas multiplicações de matrizes; assim, para o caso de eliminarmos três variáveis, com relação à complexidade temos,

$$
\begin{aligned}
\frac{m^{2}}{4} \times m \times n+ & \frac{m^{2}}{4} \times m \times 1+\frac{m^{4}}{64} \times \frac{m^{2}}{4} \times(n-1)+\frac{m^{4}}{64} \times \frac{m^{2}}{4} \times 1+\frac{m^{8}}{16384} \times \frac{m^{4}}{64} \\
& \times(n-2)+\frac{m^{8}}{16384} \times \frac{m^{4}}{64} \times 1 \\
& \frac{m^{3}}{4} \times(n+1)+\frac{m^{6}}{256} \times n+\frac{m^{12}}{1048576} \times(n-1)
\end{aligned}
$$

que teria uma complexidade de $O\left(\mathrm{~m}^{12}\right)$.

Assim, a complexidade de tempo para um sistema com $m$ inequações e $n$ variáveis é:

$$
O\left(\left(m^{3}\right)^{2^{n-2}}\right)
$$


Então, o tempo de processamento do algoritmo depende do número de variáveis $(n)$, ou seja, da dimensão do poliedro. Na Tabela 1, temos a complexidade de tempo com relação a dimensão do poliedro e na Figura 3 o gráfico desta complexidade.

Tabela 1 - Complexidade de tempo do algoritmo para o pior caso.

\begin{tabular}{cc}
$\begin{array}{c}\text { Número de } \\
\text { variáveis }\end{array}$ & $\begin{array}{c}\text { Complexidade de } \\
\text { tempo }\end{array}$ \\
\hline 2 & $O\left(m^{3}\right)^{2^{2-2}}=O\left(m^{3}\right)$ \\
3 & $O\left(m^{3}\right)^{2^{3-2}}=O\left(m^{6}\right)$ \\
4 & $O\left(m^{3}\right)^{2^{4-2}}=O\left(m^{12}\right)$ \\
5 & $O\left(m^{3}\right)^{2^{5-2}}=O\left(m^{24}\right)$
\end{tabular}

Fonte: Dados da pesquisa (2020).

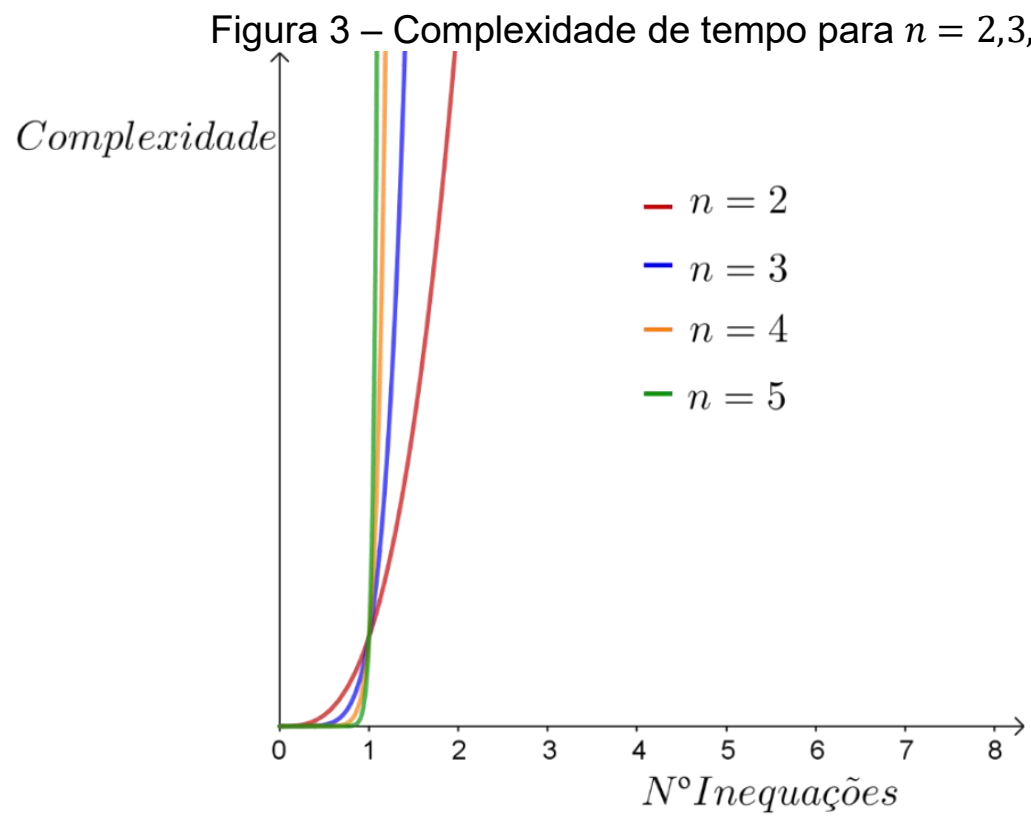

$(m)$

Fonte: Dados da pesquisa (2020).

Observando a complexidade de tempo para cada dimensão do poliedro, para $n>3$, podemos considerar o algoritmo pouco eficiente, pois o tempo de execução cresce muito.

Passemos a analisar a complexidade de espaço. Para isto, identifiquemos o quanto de memória ele precisa alocar para encontrar o ponto interior a um poliedro no pior dos casos.

Do problema geral apresentado anteriormente, precisamos de espaço em memória para alocar as seguintes matrizes, $A_{m \times n}, b_{m \times 1}, A_{\frac{m^{2}}{4} \times(n-1)}^{I^{\prime}}, b_{\frac{m^{2}}{4} \times 1}^{I}, A_{\frac{m^{4}}{64} \times(n-2)}^{I I^{\prime}}, b_{\frac{m^{4}}{64} \times 1}^{I I}, A_{\frac{m^{8}}{16384} \times(n-3)}^{I I I^{\prime}}$, $\underset{\frac{m^{8}}{16384} \times 1}{b^{I I I}}$ e $C_{\frac{m^{8}}{16384} \times \frac{m^{4}}{64}}$. Assim temos, 


$$
\begin{aligned}
S_{1}=m \times n+ & m \times 1+\frac{m^{2}}{4} \times(n-1)+\frac{m^{2}}{4} \times 1+\frac{m^{4}}{64} \times(n-2)+\frac{m^{4}}{64} \times 1 \\
+ & \frac{m^{8}}{16384} \times(n-3)+\frac{m^{8}}{16384} \times 1
\end{aligned}
$$

e

$$
S_{2}=\frac{m^{8}}{16384} \times \frac{m^{4}}{64}
$$

onde $S_{1}$ corresponde aos $A^{*}$ e $b^{*}$ dos sistemas de inequações e $S_{2}$ da matriz $C$.

Como $S_{1}$ e $S_{2}$ foram somas para três etapas, ou seja, $n=4$, para ampliar para um problema de dimensão $n$ qualquer, temos.

$$
S_{1}^{n}=\sum_{i=0}^{n-1} \frac{m^{2^{i}}}{4^{2^{i}-1}} \cdot(n+1-i)
$$

e

$$
S_{2}^{n}=\frac{m^{3.2^{n-2}}}{4^{3.2^{n-2}-2}}
$$

Assim, a complexidade de espaço para um sistema com $m$ inequações e $n$ variáveis é

$$
\sum_{i=0}^{n-1} \frac{m^{2^{i}}}{4^{2^{i}-1}} \cdot(n+1-i)+\frac{m^{3.2^{n-2}}}{4^{3.2^{n-2}-2}}
$$

A complexidade de espaço do algoritmo depende do número de variáveis $(n)$, ou seja, da dimensão do poliedro. Na Tabela 2, temos a complexidade de espaço com relação a dimensão do poliedro e, na Figura 4, o gráfico desta complexidade.

Tabela 2 - Complexidade de espaço do algoritmo.

\begin{tabular}{ccc}
$\begin{array}{c}\text { Número de } \\
\text { variáveis }\end{array}$ & \multicolumn{1}{c}{ Complexidade de espaço } \\
\hline 2 & $\sum_{i=0}^{1} \frac{m^{2^{i}}}{4^{2^{i}}-1} \cdot(3-i)+\frac{m^{3}}{4}$ & $3 m+\frac{m^{2}}{2}+\frac{m^{3}}{4}$ \\
3 & $\sum_{i=0}^{2} \frac{m^{2^{i}}}{4^{2^{i}-1}} \cdot(4-i)+\frac{m^{6}}{4^{4}}$ & $4 m+\frac{3 m^{2}}{4}+\frac{m^{4}}{32}+\frac{m^{6}}{4^{4}}$ \\
4 & $\sum_{i=0}^{3} \frac{m^{2^{i}}}{4^{2^{i}-1}} \cdot(5-i)+\frac{m^{12}}{4^{10}}$ & $5 m+m^{2}+\frac{3 m^{3}}{4^{3}}+\frac{m^{8}}{8192}+\frac{m^{12}}{4^{10}}$ \\
\hline
\end{tabular}

Fonte: Dados da pesquisa (2020). 
Figura 4 - Complexidade de espaço para $n=2,3,4$ e 5 .

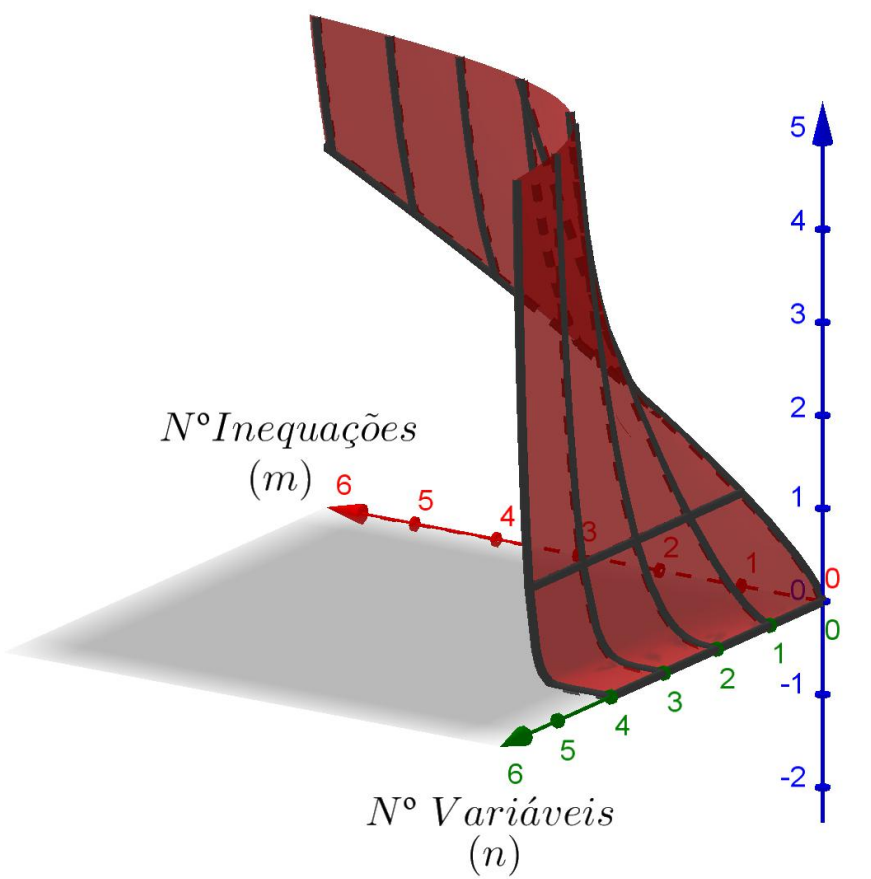

Fonte: Dados da pesquisa (2020).

Destacamos que, com o aumento do número de inequações, haverá a restrição do uso de memória computacional, bem como, com o aumento do número de variáveis $(n>3)$; portanto, não será possível obter um ponto interior utilizando o algoritmo de Fourier-Motzkin.

Em alguns casos, conforme Ajspur e Jensen (2017) apresentaram, no decorrer do processo de eliminação de Fourier-Motzkin poderão aparecer inequações redundantes adicionadas quando se aplica o método, que podem ser removidas, possibilitando a obtenção de um ponto interior. Ajspur e Jensen (2017) apresentam um algoritmo para verificar se as inequações são redundantes. Porém, para problemas maiores, mesmo eliminando as inequações redundantes, a limitação do tempo e da memória computacional impossibilita a resolução do problema.

Em contrapartida, com este método é possível identificar se o sistema $A x \leq b$ possui um conjunto solução ilimitado ou até se é incompatível.

Aplicando o algoritmo em experimentos computacionais, incluindo o método de eliminação de inequações redundantes, com o objetivo de verificar seu desempenho, geramos sistemas de inequações lineares aleatórios, cuja matriz $A$ tem dimensão $m \times n$, onde $m$ é o número de inequações e n o número de variáveis. Cada sistema corresponde a $m$ hiperplanos tangentes à esfera unitária do $R^{n}$. Para cada valor de $m$, o tempo de execução de cada rotina, apresentado nas Tabela 3 e Tabela 4, refere-se à média entre 10 experiências aleatórias realizadas para o $R^{2} \mathrm{e}$ $R^{3}$, respectivamente. 
Tabela 3 - Tempo de execução do algoritmo de pontos interiores por Fourier-Motzkin para $R^{2}$.

\begin{tabular}{cc}
\hline $\begin{array}{c}\text { Número de } \\
\text { inequações }\end{array}$ & $\begin{array}{c}\text { Tempo de } \\
\text { processamento }\end{array}$ \\
\hline 10 & 0,0131 \\
20 & 0,0134 \\
50 & 0,0146 \\
100 & 0,0156 \\
200 & 0,0199 \\
350 & 0,0327 \\
500 & 0,0608 \\
750 & 0,1662 \\
1000 & 0,2909 \\
\hline
\end{tabular}

Tabela 4 - Tempo de execução do algoritmo de pontos interiores por Fourier-Motzkin para $R^{3}$.

\begin{tabular}{cc}
$\begin{array}{c}\text { Número de } \\
\text { inequações }\end{array}$ & $\begin{array}{c}\text { Tempo de } \\
\text { processamento }\end{array}$ \\
\hline 10 & 0,0158 \\
20 & 0,0174 \\
50 & 0,0970 \\
100 & 2,1861 \\
200 & 51,1583 \\
250 & 132,0471 \\
\hline Fonte: Dados da pesquisa (2020).
\end{tabular}

Analisando os dados apresentados sobre o desempenho do algoritmo com relação ao tempo de execução, lembrando que a complexidade de tempo na realidade não representa tempo diretamente, mas o número de vezes que determinada operação considerada relevante é executada, podemos concluir que, para encontrar um ponto interior em um poliedro com $m$ inequações em 2 ou 3 variáveis, o método de Fourier-Motzkin pode ser bastante eficiente para o caso de um número pequeno de inequações. Para casos maiores, além de se tornar bem lento, em alguns casos o uso excessivo de memória pode ser restritivo.

\section{Considerações finais}

Neste trabalho, estivemos interessados em analisar o algoritmo para obter um ponto interior a um poliedro do $R^{n}$, utilizando o método de eliminação de Fourier-Motzkin. Mostramos como podemos obter este ponto, eliminando variáveis. Também apresentamos uma versão matricial deste método que facilita sua abordagem computacional, além de alguns exemplos. $O$ método mostrou-se bastante eficiente para o $R^{2}$ e o $R^{3}$ com um número pequeno de inequações, 
porém, para sistemas de inequações lineares com muitas variáveis, ou para $n>3$, pode se tornar inviável sua utilização, uma vez que o número de restrições em cada processo de eliminação de variável cresce muito rapidamente e, em alguns casos, o uso excessivo de memória torna-se restritivo, mesmo eliminando as inequações redundantes. Uma vantagem deste método é identificar se o sistema de inequações possui um conjunto solução ilimitado ou se é incompatível.

\section{Referências}

AJSPUR, Mai Lise; JENSEN, Rune Moller. Usubg Fourier-Motzkin-Elimination to Derive Capacity models of Container Vessels. IT University Thechnical Report Series, University of Copenhagen, Dinamarca, jan. 2017. Disponível em: https://en.itu.dk/research/technicalreports/technical-reports-archive/2017/tr-2017-197. Acesso em: 9 dez. 2020.

BARVINOK, Alexander; POMMERSHEIM, James E. An Algorithmic Theory of Lattice Points in Polyhedra. New Perspectives in Geometric Combinatorics, MSRI Publications, v. 38, 1999. Disponível em: https://www.maths.ed.ac.uk/ v1ranick/papers/barvpomm.pdf. Acesso em: 9 dez. 2020.

CARTIS, Coralia; Gould, Nicholas I. M. Finding a point in the relative interior of a polyhedron, with applications to compressed sensing. In: SPARTS'09 - SIGNAL PROCESSING WITH ADAPTIVE SPARSE STRUCTURED REPRESENTATIONS, Saint Malo France, 2009. Disponível em: https://citeseerx.ist.psu.edu/viewdoc/download?doi=10.1.1.703.298\&rep=rep1\&type=pdf. Acesso em: 9 dez. 2020.

CUI, Shulin; ZHANG, Shuqing; CHEN, Xuanxi; PANG, Zhenping; FU, Xiaoyang; XU, Zhang. Pointin-polyhedra test with direct handling of degeneracies. Geo-spatial Information Science, v. 14, n. 2, p. 91-97, 2011. DOI: https://doi.org/10.1007/s11806-011-0453-8.

DAHL, Geir. Combinatorial properties of Fourier-Motzkin elimination. The Electronical Jounal of linear Algebra, v. 16, p. 334-346, out. 2007. Disponível em:

https://www.elibm.org/article/10010887. Acesso em: 9 dez. 2020.

DEMMEL, James W. Applied Numerical Linear Algebra. Philadelphia: Society of Industrial and Applied Mathematics, 1997. DOI: https://doi.org/10.1137/1.9781611971446.

FOURIER, Jean-Baptiste J. Ouvress II. Paris, 1890.

LAURITZEN, Niels. Lectures on convex sets. Department Of Mathematical Sciences, University of Aarhus, Denmark, mar. 2009. Disponível em: https://www.fmf.uni-li.si/ lavric/lauritzen.pdf. Acesso em: set. 2020.

MONTICELI, André R. Um estudo sobre sistemas de inequações lineares. Orientador: Cristiano Torezzan. 2010. 118 p. Dissertação (Mestrado em Matemática) - Instituto de Matemática, Estatística e Computação Científica (IMECC), Universidade Estadual de Campinas, Campinas, SP, 2010. Disponível em:

http://repositorio.unicamp.br/bitstream/REPOSIP/306906/1/Monticeli AndreRodrigues M.pdf.

Acesso em: 9 dez. 2020.

MONTICELI, André R.; TOREZZAN, Cristiano. Método de Eliminação de Fourier-Motzkin. In: ENCONTRO REGIONAL DE MATEMÁTICA APLICADA E COMPUTACIONAL (ERMAC 2010), 1., São João del-Rei, MG, 11 a 13 nov. 2010. Anais [...]. Universidade Federal de São João del-Rei, p. 258-264, 2010. Disponível em: https://www.ufsj.edu.br/portal2-repositorio/File/iermac/anais/sessoes-tecnicas/st11.pdf. Acesso em: 9 dez. 2020. 
MOTZKIN, T. S. Beiträge zur Theorie der linearen Ungleichungen. Thesis, 1936. Reprinted in: Theodore S. Motzkin: selected papers (D. Cantor et al.), Birkhäuser, Boston, 1983. 